\title{
Aprender a usar evidência no curso de licenciatura em enfermagem: Resultados de um Focus Group
}

\author{
Óscar Ramos Ferreira', Cristina Lavareda Baixinho², \\ Marcelo Medeiros ${ }^{3}$ e Ellen Synthia Fernandes de \\ Oliveira $^{4}$ \\ ${ }^{1}$ Departamento de Fundamentos de Enfermagem, Escola Superior de Enfermagem de Lisboa, \\ Lisboa; Centro de Investigação, Inovação e Desenvolvimento em Enfermagem de Lisboa \\ (CIDNUR), ciTechaCare, Leiria, Portugal | oferreira@esel.pt | http://orcid.org/0000-0002-1703- \\ $347 \mathrm{X}$ \\ ${ }^{2}$ Departamento de Enfermagem de Reabilitação, Escola Superior de Enfermagem de Lisboa, \\ Lisboa; Centro de Investigação, Inovação e Desenvolvimento em Enfermagem de Lisboa \\ (CIDNUR), ciTechaCare, Leiria, Portugal | crbaixinho@esel.pt | http://orcid.org/0000-0001-7417- \\ 1732 \\ ${ }^{3}$ Docente titular da Faculdade de Enfermagem da Universidade Federal de Goiás, Brasil | \\ marcelo@ufg.br | https://orcid.org/0000-0001-6979-3211 \\ ${ }^{4}$ Docente do Programa de Pós-graduação em Saúde Coletiva da Universidade Federal de Goiás, \\ Brasil | ellen@ufg.br | http://orcid.org/0000-0002-0683-2620
}

Resumo: Introdução: A prática baseada na evidência tem benefícios para a melhoria dos cuidados de saúde e sustentabilidade dos sistemas de saúde. Tal como outras áreas precisa de ser aprendida. Objetivo: Compreender a perspetiva dos estudantes, do curso de licenciatura em enfermagem sobre o seu envolvimento num projeto de transferência de conhecimento para a clínica Métodos: Focus Group com oito participantes. A análise qualitativa dos achados foi efetuada com recurso ao software WebQDA® e seguiu as etapas da codificação; armazenamento/recuperação e interpretação. Seguiram-se os princípios éticos inerentes à investigação. Estudo aprovado por uma Comissão de Ética. Resultados: Emergiram quatro categorias: Pertencer, Usar evidência, Melhorar Cuidados e Desenvolver competências e respetivas subcategorias. A perceção dos estudantes é que a participação em projetos de transferência de conhecimento para a clínica gera oportunidades de aprendizagem relacionadas com o uso e comunicação de evidência, facilita a integração no serviço, a participação, o trabalho colaborativo e o desenvolvimento de competências. Conclusões: A metodologia utilizada é motivante para o estudante que é ativo na busca de evidencia e transferência do conhecimento, e que tem oportunidade de observar melhorias na prática clínica e mudanças nos contextos.

Palavras-chave: Enfermagem; Prática Clínica Baseada em Evidências; Aprendizagem; Estudantes; Gestão do Conhecimento.

\section{Learning to Use Evidence in the Nursing Degree Course: Results of a Focus Group}

Abstract: Introduction: Evidence-based practice has benefits for improving healthcare and sustainability of health systems. Like other areas needs to be learned. Goals To understand the perspective of undergraduate nursing students on their involvement in a knowledge transfer to clinical practice project Methods Focus group with eight participants. The qualitative analysis of the findings was performed using the webQDA® software and followed the steps of coding, storage/retrieval and interpretation. The ethical principles inherent to research were followed. Study approved by an Ethics Committee. Results Four categories emerged: Belonging, Using evidence, Improving care and Developing skills and their subcategories. The students' perception is that participation in knowledge transfer projects to the clinic generates learning opportunities related to the use and communication of evidence, facilitates integration into the service, participation, collaborative work and development of skills. Conclusions The methodology used is motivating for the student who is active in the search for evidence and transfer of knowledge, and who has the opportunity to observe improvements in clinical practice and changes in contexts.

Keywords: Nursing; Evidence-Based Practice; Learning; Students; Knowledge; Management. 


\section{Introdução}

A evolução da pesquisa na saúde tem trazido ganhos para a qualidade e segurança dos cuidados prestados ao cidadão, com melhoria nos indicadores e influencia nas politicas de saúde e a educação dos profissionais. Na tomada de decisão, os profissionais de saúde estão preocupados com a eficácia e viabilidade da sua abordagem e, também, com o facto da evidência e da tomada de decisão clínica ser oportuna, adequada e significativa para as pessoas ou comunidades (Apóstolo, 2017). As crenças no valor do conhecimento, a capacidade para implementar as melhores práticas, os contextos facilitadores da implementação da evidência e o apoio são alguns dos fatores facilitadores do seu uso nessa tomada de decisão (Melnyk, Gallagher-Ford, Long, \& Fineout-Overholt, 2014).

A questão da transferência da evidência tem sido debatida pela importância em assegurar uma Prática Baseada na Evidência (PBE) (Apóstolo, 2017). A transferência é mais que a divulgação ou distribuição de informação, inclui o desenvolvimento de estratégias para identificar públicos-alvo, como clínicos, gestores, 'fazedores' de políticas, consumidores, entre outros (Apóstolo, 2017). Inclui ainda o desenho, implementação e métodos para organizar e transferir a informação que é compreensível e utilizável na tomada de decisões (Apóstolo, 2017; Cardoso et al., 2020).

Todavia, os peritos observam que a adoção de uma Prática Baseada na Evidência (PBE) ainda não é um padrão dos cuidados de saúde no mundo (Melnyk et al., 2014) e alertam para a existência de uma lacuna entre a investigação, a prática, as políticas de saúde e os resultados de investigação (Oliver, Innvar, Lorenc, Woodman, \& Thomas, 2014; Apóstolo, 2017; Cardoso et al., 2021) que demoram a ser introduzidos na clínica. Os entraves à introdução da evidência são heterogêneos e prendem-se a questões metodológicas e éticas, rigor científico, capacidade de execução do projeto, dificuldades no financiamento da pesquisa, pertinência e utilidade diante das necessidades e políticas de saúde, eficácia na comunicação, na divulgação e falta de uma cultura científica de trabalho colaborativo para o desenvolvimento de produtos que promovam a introdução dos resultados nos contextos (Baixinho, \& Costa, 2019; Loura et al., 2020). Bem como pela preferência por modelos unidirecionais para a introdução dos resultados de investigação a questões relacionadas com a educação e as oportunidades dadas na formação graduada para essa aprendizagem (Cardoso et al., 2020; Cardoso et al., 2021).

A própria valorização da investigação predominantemente qualitativa também pode auxiliar na existência de uma verdadeira PBE, porque a 'transformação' qualitativa da investigação resulta numa melhor compreensão das transições e dos processos de adaptação em termos de saúde/doença, promovendo a qualidade dos cuidados, a literacia e a coresponsabilidade na gestão da saúde (Baixinho, Presado \& Ribeiro, 2019).

Apóstolo (2017) afirma que os serviços de saúde de sucesso, no futuro, serão os que investirem na procura constante de informações necessárias para orientar práticas, desenvolver líderes clínicos, bem como estabelecer e manter uma cultura de melhoria na prática diária (Apóstolo, 2017). O que reforça o exposto sobre a necessidade de investir na aprendizagem de uma PBE, o que não é feito nos bancos da escola, implicando um contacto com o contexto, conhecimento de métodos e técnicas de pesquisa e capacidade de avaliação da qualidade dos resultados obtidos, ou seja, que o estudante seja um participante ativo em alguma fase do processo de pesquisa (Cardoso et al., 2020).

Corroboramos a opinião de que dada a importância da PBE para a profissão, torna-se imperativo incutir um sentimento de paixão e entusiasmo, entre os estudantes, em relação à pesquisa e à sua relevância quotidiana para garantir resultados de saúde de qualidade (Hurlbut \& Elkins, 2018) e custo-efetivos (Watson, Sahota, Taylor, Chen, \& Lilford, 2018). 
O desenvolvimento de competências científicas implica a aquisição e a consolidação de um conjunto mínimo de atributos, conhecimentos, habilidades e atitudes, relacionadas com a PBE, o que contribui para cuidados mais seguros, de maior qualidade e centrados na pessoa (Keib et al., 2017, Loura et al., 2020). Os Ensinos Clínicos (EC) são momentos-chave para a melhoria dos conhecimentos, atitudes e competências dos alunos ao nível da PBE (Mena-Tudela et al., 2018; Kitson et al., 2018). A aprendizagem sobre investigação e utilização do conhecimento científico é maior quando é integrada nesta tipologia de ensino (Cardoso et al., 2020).

Face ao exposto, é objetivo deste estudo compreender a perspetiva dos estudantes, do curso de licenciatura em enfermagem sobre o seu envolvimento num projeto de transferência de conhecimento para a clinica.

\section{Metodologia}

Este estudo exploratório, descritivo e qualitativo, surge da questão 'Que contributos do envolvimento num projeto de transferência de conhecimento são percecionados pelos estudantes do curso de licenciatura em enfermagem?'. A escolha metodológica recaiu sobre o Fócus-Group (FG), por ser um método que possibilita a recolha de dados a um grupo, com uma experiência semelhante, permitindo a interação na discussão do grupo a partir do papel ativo do investigador na dinamização da discussão do grupo para efeitos de recolha dos dados (Silva, Veloso \& Keating, 2014).

O Protocolo do estudo cumpriu as cinco (5) fases preconizadas por Silva, Veloso e Keating, (2014): planeamento, preparação, moderação, análise dos dados e divulgação dos resultados. A revisão da literatura foi crucial para a estruturação do guião da entrevista que se organizou em torno da questão - quais os contributos do envolvimento no projeto transição segura para a vossa aprendizagem? A partir da questão inicial foi possível 'afunilar' para questões mais especificas (Krueger \& Casey, 2014). Tanto a questão central, como as secundárias, foram norteadoras, mas não tinham o intuito de condicionar a discussão, prevendo-se atempadamente que à medida que a discussão avançasse, iriam surgir questões mais específicas em função dos contributos do grupo. Esta abordagem, de compromisso com a discussão do grupo, torna assim possível aceder, por um lado, às perspetivas dos próprios participantes na primeira parte de cada discussão e, por outro, às respostas a interesses específicos do investigador na segunda parte (Silva, Veloso \& Keating, 2014).

Participaram no FG oito (8) estudantes que integraram o projeto Transição Segura entre fevereiro e julho de 2019. Foram critérios de inclusão terem realizado o último EC da sua licenciatura numa das instituições parceiras do projeto, estarem envolvidos no mesmo e manifestarem interesse em participar. Seguindo as recomendações de Krueger e Casey (2014) a amostra foi intencional e homogénea para permitir focalizar a discussão no tema, já que os participantes têm em comum uma característica relevante face ao tema em discussão - o terem participado num projeto de translação do conhecimento para a clínica.

No inicio do envolvimento no projeto os estudantes foram informados que no final poderiam participar de forma livre esclarecida no FG. Duas semanas antes foi enviado um e-mail com informação sobre os objetivos, tempo estimado de duração e identificação do moderador e co-moderador. Dado esta ser uma atividade extracurricular, creditada em suplemento ao diploma e que decorreu em simultâneo com um EC, o grupo focal só foi realizado após a saída da nota do EC. Decorreu numa sala de aulas, reservada para o efeito e que garantia as devidas condições de privacidade e gravação, bem como uma atmosfera confortável para facilitar a participação e evitar interrupções (Krueger \& Casey, 2014; Silva, Veloso \& Keating, 2014).

A opção por moderador e co-moderador baseou-se na recomendação de Kueger \& Casey (2014), bem como de Silva, Veloso \& Keating (2014) e possibilitou aumentar o rigor. 
O moderador conduziu a discussão e o co-moderador fez a gestão do equipamento de gravação, controlou as condições logísticas e do ambiente físico e tomou notas sobre a discussão (sequência das intervenções, expressões faciais, gestos, tom de voz, utilização de paralinguagem, interrupções e sobreposição de falas) (Krueger \& Casey, 2014).

Os dados gravados foram transcritos por um dos investigadores presentes de forma a permitir "visualizar" o que ocorreu no grupo (Silva, Veloso \& Keating, 2014). A análise qualitativa dos achados seguiu as etapas definidas por Bloor, Frankland, Thomas e Robson (2001): 1) codificação para a atribuição de categorias, refletindo estas os temas presentes no guião, bem como os novos, que emergiram da discussão dos grupos; 2) armazenamento/recuperação: esta fase é dedicada à compilação de todos os extratos do texto subordinados à mesma categoria de modo a poder compará-los; 3) interpretação: a partir da análise sistemática dos dados.

$\mathrm{Na}$ definição das categorias foi assegurada a representatividade, a exaustividade, a homogeneidade e a pertinência.

A análise dos achados foi feita com recurso a software informático (WebQDA®), que possibilitou a organização e análise dos achados e aumentou o rigor. A codificação dos códigos livre foi efetuada pelo investigador que fez a transcrição sendo posteriormente validada pela equipa de investigação.

Este estudo está autorizado por uma Comissão de Ética (Parecer 09/2019 HVFX). Foram respeitados os princípios éticos e formais desde a autorização dos estudantes para integrarem o projeto, enquanto atividade extracurricular, às questões éticas inerentes ao desenvolvimento da investigação. Foi assegurado também a todos os participantes 0 direito a desistir da pesquisa, o anonimato e a confidencialidade dos dados.

\section{Resultados}

Os participantes desta pesquisa foram oito estudantes, do $4^{\circ}$ ano, $2^{\circ}$ semestre, do curso de licenciatura em enfermagem, maioritariamente mulheres (07), com uma média de idades de $21,7( \pm 1,9)$ anos.

$\mathrm{Da}$ análise efetuada ao conteúdo do FG emergiram 190 unidades de registo, organizadas em quatro categorias: Pertencer, Usar evidência, Melhorar Cuidados e Desenvolver competências e respetivas subcategorias (Tabela 1).

Tabela 1. Corpus da análise de conteúdo. Lisboa; Portugal. 2021.

\begin{tabular}{|c|c|c|}
\hline Categoria & Subcategoria & UR \\
\hline \multirow[t]{3}{*}{ Pertencer } & Integração & 10 \\
\hline & Participação & 13 \\
\hline & Subtotal & 48 \\
\hline Usar & Oportunidades de Aprendizagem & 32 \\
\hline \multirow[t]{3}{*}{ Evidência } & Aprender Evidência & 44 \\
\hline & Comunicar Evidência & 15 \\
\hline & Subtotal & 114 \\
\hline Melhorar & Oportunidades de melhoria & 10 \\
\hline \multirow[t]{2}{*}{ cuidados } & Promover melhores cuidados & 10 \\
\hline & Subtotal & 20 \\
\hline \multirow{3}{*}{ Competências } & Liderança & 1 \\
\hline & & 8 \\
\hline & Total & 190 \\
\hline
\end{tabular}


A perceção dos estudantes é que um dos grandes contributos do seu envolvimento é a facilidade de integração no serviço, pela existência de um projeto e de um trabalho continuo entre escola e serviço, onde outros colegas já estiveram envolvidos: 'ajudou também a integrar na equipa, por isso, foi muito positivo, sim' (P4); 'o facto de saber que no projeto o trabalho que eu estou a desenvolver será importante também mais para a frente numa fase posterior foi bom para a integração' (P8)

A integração bem sucedida possibilita uma maior participação do estudante em atividades, nas reuniões e nas decisões relativas ao projeto e até das atividades para concretizar o seu EC 'o estar disponível para responder às nossas questões e às nossas dúvidas, sempre OK, sempre a explicar como funciona e como fazem aqui, também a partilha das nossas próprias ideias, o que achávamos que poderia ser feito, partilharmos estas ideias connosco e estarem acessíveis para falar e partilhar isso connosco também acho que é muito importante', (P1); 'nós tivemos a possibilidade de participar numa consulta de vida ativa e no final a enfermeira até pediu a nossa opinião sobre coisas que nós pudéssemos lembrar que eles não, talvez eles não se lembrariam, até sabe bem estarmos envolvidos e contarem connosco no serviço' (P8); 'os enfermeiros tiveram de ter essa abertura para que nós pudéssemos participar (...)ela própria me incentivava e dava-me feedback' (P7); 'até porque somos estudantes há muita coisa que não participamos nos serviços e assim acaba por ser dada possibilidade' (P3).

Esta metodologia cria oportunidades de trabalho colaborativo com os enfermeiros e outros elementos da equipa multidisciplinar: 'foi uma das coisas que eu também senti, apesar de estar a apresentar para enfermeiros coordenadores de unidade e enfermeiros chefes, psicólogo e equipa multidisciplinar o facto foi que senti que eles não me tratavam só como um aluno' (P7), 'eu considero que por parte da equipa nas reuniões multidisciplinares nós, enquanto estudantes, podíamos ter um papel ativo nessas reuniões e nem sempre acontece, não agora, mas nos estágios anteriores, mesmo quando assistíamos a formações em serviço, geralmente não participávamos, nem com dúvidas, foi uma mais valia' (P3).

A integração e as oportunidades de participação e trabalho colaborativo geram no estudante um sentimento de pertença, até porque 'Essa proximidade também permitiu aprender outras coisas para além do projeto' (P7).

Em relação à categoria Usar Evidência o fato de o projeto ter como finalidade a introdução da evidência na clínica proporciona oportunidades de aprendizagem paralelas e complementares aos resultados esperados para o EC 'teríamos já de desenvolver uma monografia, mas só que o fato de sabermos que estávamos a trabalhar para o projeto ... e então colocou em mim a pressão ah! OK! então isto tem de ser mesmo alguma coisa com uma base científica muito forte, para além, daquilo que já seria de esperar para a monografia' (P1), 'A mais valia dos projetos é de facto esta, o vermos o ideal e as dificuldades do contexto em pensarmos fora da caixa como resolver, o que nós aprendemos e como podemos aplicar no contexto'(P2), 'deixei de aceitar tudo como certo ou correto, quando me dizem faz-se assim pergunto sempre o porquê e baseado em quê' (P1).

A Aprendizagem da evidência processa-se de modo articulado 'Constatar que um trabalho teórico pode ser implementado na prática e dá gosto esta parte dos resultados que obtivemos com a revisão integrativa da literatura poderem ser realmente utilizados e não ser só um trabalho' (P5); ' $E$ depois conseguíamos ver também que mesmo que não haja muita investigação feita naquela área poderemos ser nós próprios, um dia mais tarde, quem sabe, a produzir informação e mais conhecimento e, desenvolver mais o que trabalhámos agora, acaba por ser um contributo' (P1); 'temos também outras hipóteses de colher e analisar dados do nosso contexto, e assim aprendemos algumas coisas da investigação' (P7); 'Para mim ajudou a diminuir as diferenças entre a teoria e a prática, isto parece estranho de se dizer, mas, muitas vezes criticamos que os conteúdos da escola são muito teóricos e pouco aplicados na prática, há diferenças grandes, mas esta experiência permitiu agarrar na teoria e aplicar na prática que é algo que não fizemos nos outros EC' (P8). 
A aprendizagem sobre evidência também se consegue pelas oportunidades dadas aos estudantes na comunicação de resultados dos projetos 'Sim e a mais pertinente, acabou por ser um desafio quer de escrita, quer também de comunicação oral, porque passar de um trabalho muito....e compactá-lo todo numa única folha, passo a expressão, é difícil' (P6), 'E não é só isso, é termos aquela noção que nós nos pósteres na universidade vão ser expostos aos professores, vão ser expostos aos colegas, e desta vez vai ser exposto ao público e isso dá outro peso, digamos assim, ao trabalho que estamos a elaborar e outra responsabilidade, é um trabalho que não é só nosso, colaboramos nele, temos de ter mais brio, mais rigor, e maior noção do que estamos a escrever' (P7).

Para os participantes o uso da evidência possibilita 'Melhorar cuidados' porque 'não era só a prestação de cuidados aos idosos era também o termos um projeto macro que permitia enquadrar esses cuidados na segurança e qualidade, tínhamos a prestação de cuidados e um projeto extra, mas que se relacionavam' (P8), 'A consulta de follow up da unidade vai permitir, futuramente, não já agora, mas futuramente, mudar o seguimento destes doentes, ter feedback da evolução até para se melhorar as práticas na unidade e poder acompanhar durante mais tempo estes doentes, saber o que o doente apresenta depois do internamento e mantê-lo ligado à unidade é algo bastante prático' (P3).

$\mathrm{Na}$ categoria Desenvolver Competências emergem aspetos relacionados com a autonomia, a gestão do tempo e conflitos 'no meu caso não houve assim grandes conflitos, mas é sempre uma possibilidade e algo que acaba por ser desenvolvido' (P7), 'quando encontrávamos um problema tínhamos de o resolver e sim foi como tu dizias (referindo-se ao desenrascar) principalmente nessa área' (P8). Um participante salienta ainda a possibilidade de desenvolver competências de liderança 'em que uma pessoa, digamos assim, tem de distribuir trabalho para as pessoas trabalharem de alguma forma equitativamente, digamos assim , mas há sempre uma pessoa que acaba por coordenar as coisas'(P7).

\section{Discussão}

A análise da participação destes estudantes no FG permite compreender a importância atribuída ao pertencer à equipa e que na sua opinião é facilitada pela integração nos projetos em curso na instituição. A 'Pertença' é uma característica universal do ser humano e é uma necessidade humana básica (Sedgwick, \& Yonge, 2008). Ashktorab, Hasanvand, Seyedfatemi, Salmani, e Hosseini (2017) afirmam que esta é uma das necessidades mais importantes dos estudantes para trabalharem de forma fiável no ambiente clínico.

A compreensão e o reconhecimento desta característica distintiva pode reduzir o stress e a tensão (Ashktorab, et al., 2017) e promover a aprendizagem. Assim, os enfermeiros orientadores devem ajudar os estudantes a tornarem-se membros da equipa e fomentar o desenvolvimento do sentimento como se pertencessem, construindo pontes entre o pessoal e os estudantes (Sedgwick, \& Yonge, 2008). Para Ditzel (2017) o desenvolvimento do sentimento de pertença a uma comunidade envolve quatro elementos: a adesão, a influência, a integração e satisfação de necessidades e a ligação emocional partilhada (Ditzel, 2017). Assim entendemos que investigações futuras devem explorar os achados deste estudo e a relação com os elementos referidos por Ditzel (2017), tanto mais que os resultados obtidos apontam para que a existência de projetos comuns entre escola e serviços, bem como o continuo envolvimento de estudantes promove a integração, cria oportunidades de participação e trabalho colaborativo que aumenta o sentimento de pertença. Por outro lado, a metodologia utilizada torna o estudante ativo na busca de evidencia e transferência do conhecimento, reforçando o sentimento de pertença e que fazem parte da equipa quando são conhecidos pessoal e profissionalmente (Sedgwick, \& Yonge, 2008; Cardoso et al., 2021).

$\mathrm{Na}$ categoria usar evidência os participantes reforçam a importância de aprenderem como se transfere a evidência e até como isso ajuda na diminuição do fosso entre a teoria e a prática. 
No debate acadêmico e público sobre o tema a transferência do conhecimento para a clínica não é um assunto propriamente recente. Embora ultimamente, a transferência do conhecimento para a clínica, tenha ganho notoriedade, os investigadores têm sido confrontados com desafios complexos, desde o desenho até a utilização dos resultados dos seus estudos, o que não possibilita a sua apropriação pela práxis (Cardoso et al., 2020; Cardoso et al., 2021). Nem também pelos estudantes de enfermagem, dado que $87 \%$ não usa os resultados da investigação na clínica e referem que a não utilização da evidência disponível relaciona-se com conhecimento insuficiente sobre a utilização da investigação (Ertug, \& Önal, 2014).

Observamos no discurso dos participantes que há um hiato entre a teoria e a prática na tomada de decisão clínica. Este achado encontra eco na voz dos autores que reclamam uma rutura paradigmática com esta visão de um vazio entre dois mundos (teórico e prático). Para tal torna-se necessário promover a reflexão sobre as práticas, os "modos de fazer" e as consequências da atividade, usando o conhecimento baseado na investigação, para a tomada de decisão (Baixinho, \& Costa, 2019). Para tal, acredita-se que os docentes não só devem instruir os estudantes, mas também criar oportunidades de participação em investigação (Ertug, \& Önal, 2014, Loura et al., 2020).

Face à análise do conteúdo deste $F G$ podemos referir que o envolvimento dos estudantes em projetos de transferência para a clínica pode auxiliar na resolução do problema. Não só pela aprendizagem de como transferir conhecimento para a clínica, mas também pelo papel futuro que, estes recém-licenciados, podem ter nas suas equipas, na divulgação das suas aprendizagens e na criação de oportunidades para o debate/reflexão sobre o uso e transferência da evidência.

Como os próprios estudantes percecionam o envolvimento no projeto e a aprendizagem de como usar a evidência tem impactos na melhoria dos cuidados e no desenvolvimento de outras competências transversais. O que reforça as recomendações dos autores para uma articulação ativa entre as instituições académicas e as clinicas criando oportunidades de trabalho colaborativo que possam beneficiar o desenvolvimento do estudante e simultaneamente contribuir para a melhoria das práticas (AIThiga, Mohidin, Park, \& Tekian, 2017; Cardoso et al., 2020; Cardoso et al., 2021).

Uma última nota, nesta discussão, para ressalvar o espírito critico e reflexivo que estão explícitos em algumas narrativas dos participantes e que se estende à recomendação para a manutenção do projeto e para a participação dos estudantes no mesmo.

\section{Conclusões}

Os resultados deste estudo permitem compreender os contributos do envolvimento dos estudantes em projetos de transferência do conhecimento para a clinica. Emergem na análise de conteúdo categorias: Pertencer, Usar evidência, Melhorar Cuidados e Desenvolver competências. A categoria com maior expressão é Usar evidência, com 114 das 190 unidades de registo obtidas no Focus Group.

Face aos resultados recomenda-se que nos ensinos clínicos os estudantes tenham oportunidade de utilizar os resultados de investigação e que aprendam a transferi-los para os settings clínicos. Cremos que esta será uma competência core para os profissionais de saúde do futuro, com impacto na melhoria dos cuidados e na sustentabilidade dos sistemas de saúde.

A limitação do estudo prende-se com o método e com a possível influência da interação do grupo na resposta individual. Recomenda-se que estudos futuros recorram à entrevista individual, em profundidade, para uma maior compreensão do 'Pertencer' nas oportunidades de 'Aprender evidência'. 


\section{Agradecimentos}

Agradecemos às Instituições Parceiras as condições criadas para o desenvolvimento deste projeto e em particular aos estudantes envolvidos pelo seu contributo.

\section{Referências}

AlThiga, H., Mohidin, S., Park, Y.S., \& Tekian, (2017). A. Preparing for practice: Nursing intern and faculty perceptions on clinical experiences. Medical Teacher. 39(sup1):S55-S62. https://doi.org/10.1080/0142159X.2016.1254739.

Apóstolo, J. (2017). Síntese da evidência no contexto da translação da ciência. Coimbra, Portugal: Escola Superior de Enfermagem de Coimbra (ESEnfC).

Ashktorab, T., Hasanvand, S., Seyedfatemi, N., Salmani, N., \& Hosseini, S. V. (2017). Factors Affecting the Belongingness Sense of Undergraduate Nursing Students towards Clinical Setting: A Qualitative Study. Journal of caring sciences, 6(3):221-235. https://doi.org/10.15171/jcs.2017.022

Baixinho, C.L., \& Costa, A.P. (2019). From the hiatus in the theory - practice discourse to the clinic based on the uniqueness of knowledge. Esc Anna Nery; 15;23(3):e20190141. http://dx.doi.org/10.1590/2177-9465-ean-2019-0141

Baixinho, C.L., \& Presado, M.H., Ribeiro, J. (2019). Qualitative research and the transformation of public health. Ciência \& Saúde Coletiva; 24 (5)1583-1583. https://doi.org/10.1590/141381232018245.05962019

Cardoso, M., Baixinho, C.L., Ferreira, Ó., Nascimento, P., Pedrosa, R., \& Gonçalves, P. (2020). Autopercepção dos estudantes sobre a participação em atividades extracurriculares de transferência de conhecimento: o exemplo da Transição Segura. New Trends on Qualitative Research. 2(2020):588-601. https://doi.org/10.36367/ntqr.2.2020.588-601

Cardoso, M., Baixinho, C.L., Ferreira, Ó., Nascimento, P., Pedrosa, R., \& Gonçalves, P. (2021). Aprender prática baseada na evidência pelo envolvimento em atividades de investigação autopercepção dos estudantes. Cogitare enfermagem. 26:e79806. http://dx.doi.org/10.5380/ce.v26i0.79806

Ditzel, L.M. (2017). Sense of Community among Nurses: Results of a Study. International Journal of Studies in Nursing; 2(2). https://doi.org/10.20849/ijsn.v2i2.191

Ertug, N., \& Önal, H. (2014). Undergraduate Nursing Students' Research Activities and Utilization: A Turkish Sample. Aquichan, 14(2):251-260. https://doi.org/10.5294/aqui.2014.14.2.11

Ferreira, E.M., Lourenço, O.M., Costa, P.V., et al. (2019). Active Life: a project for a safe hospitalcommunity transition after arthroplasty. Revista Brasileira de Enfermagem, 72(1):147-153. doi: https://dx.doi.org/10.1590/0034-7167-2018-0615

Hurlbut, J., \& Elkins, M. (2018). Redesigning an Undergraduate Nursing Research Course Using Innovative Teaching Strategies. SM J Nurs; 4(1):1017. Available from: https://smjournals.com/nursing/fulltext/smjn-v4-1017.pdf

Keib, C.N., Cailor, S.M., Kiersma, M.E., \& Chen, A.M.H., 2017. Changes in nursing students'perceptions of research and evidence-based practice after completing a research course. Nurse Education Today. 54:37-43. https://doi.org/10.1016/j.nedt.2017.04.007.

Kitson, A., Brook, A., Harvey, G., Jordan, Z., Marshall, R., O’Shea, R., \& Wilson, D. (2018). Using complexity and network concepts to inform healthcare knowledge translation. International Journal of Health Policy and Management, 7(3):231-243. https://doi.org/10.15171/IJHPM.2017.79

Krueger, R.A. \& Casey, M.A. (2014). Focus Groups: A Practical Guide for Applied Research (5th ed.). New Delhi: Sage.

Loura, D., Bernardes, R., Baixinho, C.L., Rafael, H., Félix, I., \& Guerreiro, M. (2020). Aprender em projetos de investigação durante a licenciatura em enfermagem: revisão integrativa da literatura. New Trends on Qualitative Research. 3(2020):293-304.

https://doi.org/10.36367/ntqr.3.2020.293-304 
Melnyk, B., Gallagher-Ford, L., Long, L., \& Fineout-Overholt, E. (2014). The establishment of evidence-based practice competencies for practicing registered nurses and advanced practice nurses in real-world clinical settings: proficiencies to improve healthcare quality, reliability, patient outcomes, and costs. Worldviews on Evidence-Based Nursing, 11(1):5-15. http://dx.doi.org/10.1111/wvn.12021

Mena-Tudela, D., González-Chordá, V.M., Cervera-Gasch, A., Maciá-Soler, M.L., \& Orts-Cortés, M. I. (2018). Effectiveness of an Evidence-Based Practice educational intervention with secondyear nursing students. Rev Latino-Am Enfermagem, 26:e3026. https://dx.doi.org/10.1590/1518-8345.2502.3026

Oliver, K., Innvar, S., Lorenc, T., Woodman, J., \& Thomas, J. (2014). A systematic review of barriers to and facilitators of the use of evidence by policymakers. BMC Health Services Research, 14, 2. http://dx.doi.org/10.1186/1472-6963-14-2

Sedgwick, M.G., \& Yonge, O. (2008). 'We're it', 'we're a team', 'we're family' means a sense of belonging. Rural Remote Health; 8(3):1021. Epub 2008 Sep 4. PMID: 18771338.

Silva, I.S., Veloso, A.L., \& Keating, J.B. (2014). Focus group: Considerações teóricas e metodológicas. RLE; 26(26):175-90. Available from: https://revistas.ulusofona.pt/index.php/rleducacao/article/view/4703

Watson, S. I., Sahota, H., Taylor, C. A., Chen, Y. F., \& Lilford, R. J. (2018). Cost-effectiveness of health care service delivery interventions in low and middle income countries: a systematic review. Global health research and policy, 3, 17 https://doi.org/10.1186/s41256-018-0073-z 